\title{
Index et charge glycémiques de quatre jus de fruits chez des sujets adultes jeunes apparemment sains
}

\author{
Moutawakilou GOMINA ${ }^{1,4^{*}}$, Gwladys SANT'ANNA ${ }^{1}$, Tarik SALIFOU ${ }^{1}$, \\ Adebayo ALASSANI ${ }^{2}$, Ibrahim MAMA CISSE ${ }^{3}$, Lamine BABA-MOUSSA ${ }^{4}$ et \\ Simon AKPONA ${ }^{5}$ \\ ${ }^{1}$ UER de Biochimie, Faculté de Médecine, Université de Parakou, Bénin. \\ ${ }^{2}$ UER de Médecine Interne, Faculté de Médecine, Université de Parakou, Bénin. \\ ${ }^{3}$ UER de Santé au Travail, Faculté de Médecine, Université de Parakou, Bénin. \\ ${ }^{4}$ Laboratoire de Biologie et Typage Moléculaire en Microbiologie, Université d'Abomey-Calavi, Bénin. \\ ${ }^{5}$ Laboratoire de Biologie Clinique, Clinique Espace Santé Ste Elisabeth, Parakou, Bénin. \\ *Auteur correspondant ; E-mail: elboutraguero@yahoo.fr
}

\author{
Received: 21-08-2021
}

Accepted: 14-12-2021

Published: 31-12-2021

\section{RESUME}

La consommation de jus de fruits naturels n'a pas d'effet significatif ni sur la glycémie à jeun ni sur le contrôle glycémique chez les diabétiques. L'objectif de cette étude était de déterminer l'index et la charge glycémiques de quatre jus de fruits chez des sujets adultes jeunes apparemment sains. Il s'agissait d'une étude quasi expérimentale, qui a inclus 32 sujets adultes volontaires apparemment sains, sélectionnés selon les critères de l'Organisation Mondiale de la Santé et répartis en 4 groupes de 8 . Chaque groupe de sujets a consommé à deux reprises l'aliment de référence (pain blanc pour 2 groupes; glucose pour les 2 autres), puis une portion de l'un des jus de fruits contenant une quantité équivalente à $25 \mathrm{~g}$ de glucides. L'Index Glycémique (IG) et la Charge Glycémique (CG) ont été déterminés par la méthode standard. Le jus DAFANI orange-mangue avait un IG faible $(47,50)$, les jus DAFANI cocktail mangue-ananas-fruit de la passion $(62,25)$ et ananas IRA $(64,12)$ un IG moyen, et le jus DAFANI nectar mangue $(87,62)$ un IG élevé. Tous les jus de fruits avaient une CG modérée (comprise entre 11 et 19). Bien que la CG des jus de fruits étudiés soit modérée, leur consommation doit être contrôlée en particulier chez les diabétiques.

(C) 2021 International Formulae Group. All rights reserved.

Mots clés : Index glycémique, charge glycémique, jus de fruits, Bénin.

\section{Glycemic index and glycemic load of four fruit juices in apparently healthy young adults' subjects}

\begin{abstract}
Consuming natural fruit juices has no significant effect on either fasting blood sugar or blood sugar control in diabetics. This study was aimed to determine the glycemic index and glycemic load of four fruit juices in apparently healthy young adult subjects. Through a quasi-experimental study, 32 apparently healthy adult volunteers were selected using the World Health Organization criteria, and divided into 4 groups of 8. Each group of subjects consumed twice the reference food (white bread for 2 groups; glucose for the 2 other), then a serving
\end{abstract}


of one of the fruit juices containing an amount equivalent to $25 \mathrm{~g}$ of carbohydrate. The Glycemic Index (GI) and Glycemic Load (GL) was determined by the standard method. DAFANI orange-mango juice had a low GI (47.50), DAFANI mango-pineapple-passion fruit cocktail (62.25) and pineapple IRA (64.12) juices had an average GI, and DAFANI mango nectar juice (87.62) a high GI. All fruit juices had a moderate GL (between 11 and 19). Despite the moderate GL of fruit juices studied, their consumption must be controlled, particularly in diabetics.

(C) 2021 International Formulae Group. All rights reserved.

Keywords: Glycemic index, glycemic load, fruit juice, Benin.

\section{INTRODUCTION}

Le diabète sucré est une maladie chronique non transmissible et l'une des principales causes de mortalité et de morbidité dans le monde. L'obésité, la sédentarité, le régime alimentaire surtout centré sur l'alimentation occidentale et l'hérédité sont autant de facteurs favorisant du diabète (Makoutode et al., 2017). L'alimentation a donc un impact considérable dans l'augmentation de la prévalence de cette maladie chronique (Kimokoti et Millen, 2016) et constitue ainsi un aspect crucial dans la gestion globale du diabète. Ainsi, la diète prescrite aux diabétiques doit tenir compte non seulement de l'apport calorique mais aussi de la capacité des aliments à induire une réponse glycémique élevée ou basse (Madhu, 2017).

Certains paramètres tels que l'Index Glycémique (IG) et la Charge Glycémique (CG) permettent d'apprécier la réponse glycémique des aliments. Si l'IG est un critère de classement des aliments contenant des glucides basé sur leurs effets sur la glycémie durant les deux heures suivant leur ingestion, la $\mathrm{CG}$, mesure qui implique la quantité et la qualité des glucides alimentaires, permet de mieux apprécier le pouvoir hyperglycémiant des aliments (Augustin et al., 2015). Ces notions d'IG et CG sont valables aussi bien pour les fruits que les jus de fruits et les autres types d'aliments.

Le jus de fruit est obtenu à partir de fruits sains et mûrs, frais ou conservés par le froid, d'une espèce ou de plusieurs espèces en mélange. Les fruits, en raison de leur richesse en fibres, antioxydants et composés phytochimiques qui peuvent avoir des effets bénéfiques sur la santé, sont recommandés pour la prévention primaire du diabète de type 2 (Muraki et al., 2013). En revanche, les travaux indiquant que les jus de fruits possèdent des effets protecteurs attribués aux fruits entiers ne sont toujours pas concluants (Pereira et Fulgoni, 2010). La consommation des jus de fruits est moins souhaitable car ils contiennent moins de fibres alimentaires que les fruits entiers et apportent des calories supplémentaires lorsque consommés en excès en raison d'un ajout de sucre (Wang et al., 2008). Plusieurs études ont montré qu'il n'y avait pas d'effet significatif des jus de fruits (100\% jus d'orange frais, nectar de jus d'orange sucré) sur la glycémie à jeun (Murphy et al., 2017; Papandreou et al., 2019), ni sur le contrôle glycémique (Xi et al., 2014). Selon une revue systématique, il n'y avait pas d'association significative entre la consommation de fructose et le risque de survenue du diabète de type 2 (Tsilas et al., 2017).

Au Bénin, de nombreuses variétés de jus de fruits, de fabrication locale ou importées, sont consommées par les populations. Si de rares études ont abordé la réponse glycémique induite par la consommation de boissons alcoolisées locales (Gomina Assoumanou et al., 2011), il n'existe presque pas de travaux portant sur l'impact de ces jus sur la glycémie. Ce travail avait pour objectif de déterminer l'index et la charge glycémiques de quatre jus de fruits, commercialisés au Bénin, chez des sujets adultes jeunes apparemment sains, afin de contribuer à l'amélioration de la diététique des populations en particulier des diabétiques. 


\section{MATERIEL ET METHODES}

\section{Type et période d'étude}

Il s'agissait d'une étude interventionnelle, de type quasi expérimental, dont la collecte des données a couvert la période du $1^{\text {er }}$ février au 31 mars 2020.

\section{Population d'étude}

L'étude concernait les sujets adultes jeunes (âge moyen: 23,18 $\pm 02,03$ ans), apparemment sains, âgés de 18 à 30 ans, dont le bilan biochimique de base (glycémie à jeun, créatininémie, ASAT et ALAT) était normal et ayant consenti librement d'y participer. Les fumeurs, les sujets sous traitement médicamenteux ou diététique en cours, hypertendus, ayant un antécédent de diabète sucré, présentant un trouble de la crase sanguine, souffrant de pathologies hépatiques, pancréatiques, intestinales ou de troubles de la glycorégulation, et les femmes enceintes n'y ont pas été inclus. Au total 32 sujets des deux sexes ont été recrutés et répartis en 4 groupes (G1, G2, G3, G4) de 8 sujets en utilisant les recommandations sur l'expérimentation pour la réponse glycémique (FAO/WHO, 1998).

\section{Aliment de référence}

Une charge de $25 \mathrm{~g}$ d'aliment de référence a été utilisée au lieu de $50 \mathrm{~g}$ (Brouns et al., 2005), afin d'éviter de faire ingérer de grandes quantités de jus de fruits aux sujets. Ainsi les aliments de références utilisés étaient: le glucose (dextrose anhydrous purified LOBA Chemie LOT SL 29541112) et le pain blanc.

\section{Aliments tests}

Il s'agissait de quatre jus de fruits commercialisés au Bénin, acquis dans les supermarchés. Leurs valeurs nutritives, inscrites sur les emballages, sont présentées dans le Tableau 1.

\section{Déroulement de l'expérimentation}

Un régime normo glucidique, une activité physique normale 72 heures avant le début de l'expérimentation, et un jeûne de 8 heures d'horloge au moins la veille de l'expérimentation ont été conseillés aux sujets.

\section{Consommation de l'aliment de référence}

Le jour de l'expérimentation, les sujets ont été admis dans la salle prévue à cet effet à 8 heures du matin. Après un repos de 15 minutes, un cathéter veineux a été mis en place au niveau de la veine cubitale et l'échantillon de sang prélevé sur tube oxalate-fluorure de sodium pour la glycémie de base T0. Les sujets des groupes G1 et G2 ont consommé $25 \mathrm{~g}$ de glucose dilué dans $250 \mathrm{~mL}$ d'eau plate ; ceux des groupes $\mathrm{G} 3$ et $\mathrm{G} 4,50 \mathrm{~g}$ de pain blanc (correspondant à $25 \mathrm{~g}$ de glucides) accompagné de $250 \mathrm{~mL}$ d'eau plate ; la durée de la consommation des aliments était de 10 minutes. Le prélèvement de sang veineux a été poursuivi à $\mathrm{T} 15, \mathrm{~T} 30, \mathrm{~T} 45, \mathrm{~T} 60, \mathrm{~T} 90$ et T120 minutes.

\section{Consommation des jus de fruits}

Pour les jus de fruits étudiés, le même processus que celui de l'aliment de référence a été suivi, avec la consommation des quantités suivantes: G1 (DAFANI cocktail mangue et orange : 190,83 mL); G2 (jus d'ananas IRA : 192,3 mL); G3 (DAFANI cocktail mangue, ananas, fruit de la passion : 186,56 mL) ; G4 (DAFANI nectar de mangue: $188 \mathrm{~mL}$ ). L'aliment de référence a été expérimenté en deux séances et les fruits testés en une seule séance. Les séances ont été séparées d'au moins une semaine d'intervalle.

\section{Dosage de la glycémie}

Les échantillons de sang prélevés ont été centrifugés à 4000 tours par minute pendant 5 minutes pour obtenir les plasmas sur lesquels le dosage du glucose a été effectué par la méthode enzymatique en point final à la glucose oxydase-peroxydase en utilisant les kits Elitech Group sur automate SELECTRA PRO S.

\section{Analyse des données}

L'analyse des données a été faite à l'aide du logiciel SPSS statistics version 26 (IBM corps, 2019). Les variables quantitatives ont été exprimées en moyenne \pm écart-type. 
L'IG des jus de fruits a été déterminé par la méthode des trapèzes (Brouns et al., 2005). La CG a été calculée par la formule de Monro et Shaw (2008). Les critères de Foster-Powell et al. (2002) ont été utilisés pour classifier l'IG et la CG.

\section{Aspects éthiques}

Le protocole de recherche de cette étude a obtenu l'approbation du Comité Local d'Ethique pour la Recherche Biomédicale de l'Université de Parakou (avis nº 0297/CLERB$\mathrm{UP} / \mathrm{P} / \mathrm{SP} / \mathrm{R} / \mathrm{SA}$ ).

Tableau 1: Valeurs nutritives des jus de fruits testés pour $100 \mathrm{~mL}$ (source: étiquettes des jus de fruits).

\begin{tabular}{lccc}
\hline & Energie (kcal) & Glucides (g) & Fibres (g) \\
\hline Jus d'ananas IRA & 44,57 & 13,0 & 14 \\
$\begin{array}{l}\text { DAFANI cocktail mangue-ananas-fruit } \\
\text { de la passion }\end{array}$ & 55 & 13,4 & $\mathrm{NP}$ \\
$\begin{array}{l}\text { DAFANI nectar mangue } \\
\text { DAFANI cocktail mangue-orange }\end{array}$ & 55 & 13,3 & $\mathrm{NP}$ \\
\hline \multicolumn{1}{c}{ NP : non précisé. } & 54 & 13,1 & $\mathrm{NP}$ \\
\end{tabular}

\section{RESULTATS}

\section{Variation de la glycémie pendant deux} heures après la consommation de chacun des quatre fruits

La réponse glycémique du jus DAFANI cocktail orange-mangue était inférieure à celle du glucose pris comme aliment de référence. Un pic glycémique a été observé à T30 minutes après la consommation aussi bien du jus DAFANI cocktail orange-mangue que du glucose (Figure 1A). Le jus d'ananas IRA a induit une réponse glycémique presque superposable à celle du glucose, l'aliment de référence (Figure 1B). Toutefois, le pic glycémique a été observé à T30 minutes pour le jus d'ananas IRA et à T45 minutes pour le glucose. La réponse glycémique du jus DAFANI cocktail mangue-ananas et fruit de la passion (MAP) était superposable à celle du pain de T0 à T30 minutes, mais était devenue inférieure de T30 à T120 minutes. Un pic glycémique a été observé à T30 minutes pour le jus DAFANI cocktail MAP et à T45 minutes pour le pain, l'aliment de référence du groupe (Figure 1C). Le jus DAFANI nectar mangue avait induit une réponse glycémique superposable à celle du pain de $\mathrm{T} 0$ à $\mathrm{T} 30$ minutes, et inférieure de T30 à T120 minutes. Les deux aliments ont culminé à T30 (Figure 1D).

\section{Index et charge glycémiques des fruits}

Les index et charge glycémiques variaient en fonction du jus testé (Tableau 2). Le jus DAFANI orange-mange avait un index glycémique faible $(\leq 55 \%)$; les jus d'ananas IRA et DAFANI cocktail MAP un index glycémique moyen (56\% à 69\%); le jus DAFANI nectar mangue un index glycémique élevé ( $\geq 70 \%$ ). Tous les jus de fruits avaient une charge glycémique modérée (comprise entre 11 et 19$)$. 


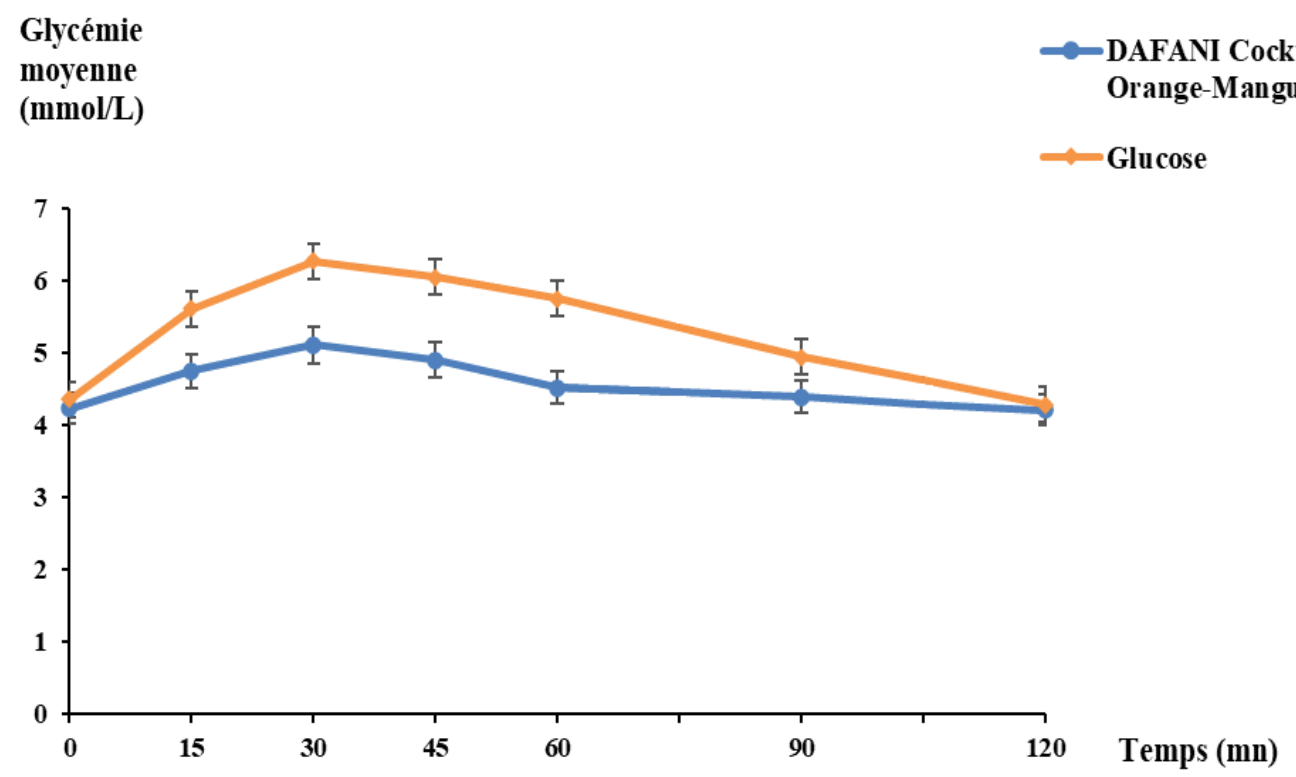

Figure 1A : Groupe 1 (Jus DAFANI Cocktail orange-mangue).

\section{Glycémie}

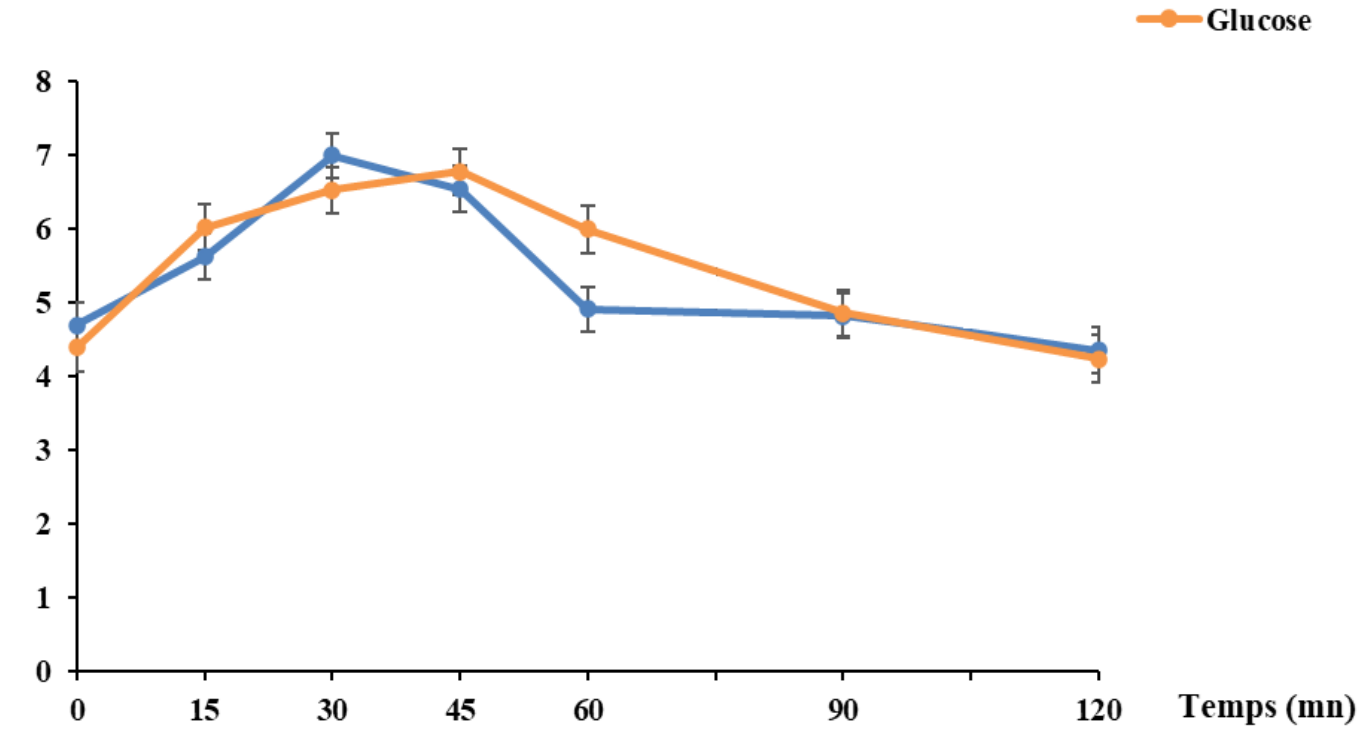

Figure 1B : Groupe 2 (Jus d'ananas IRA). 


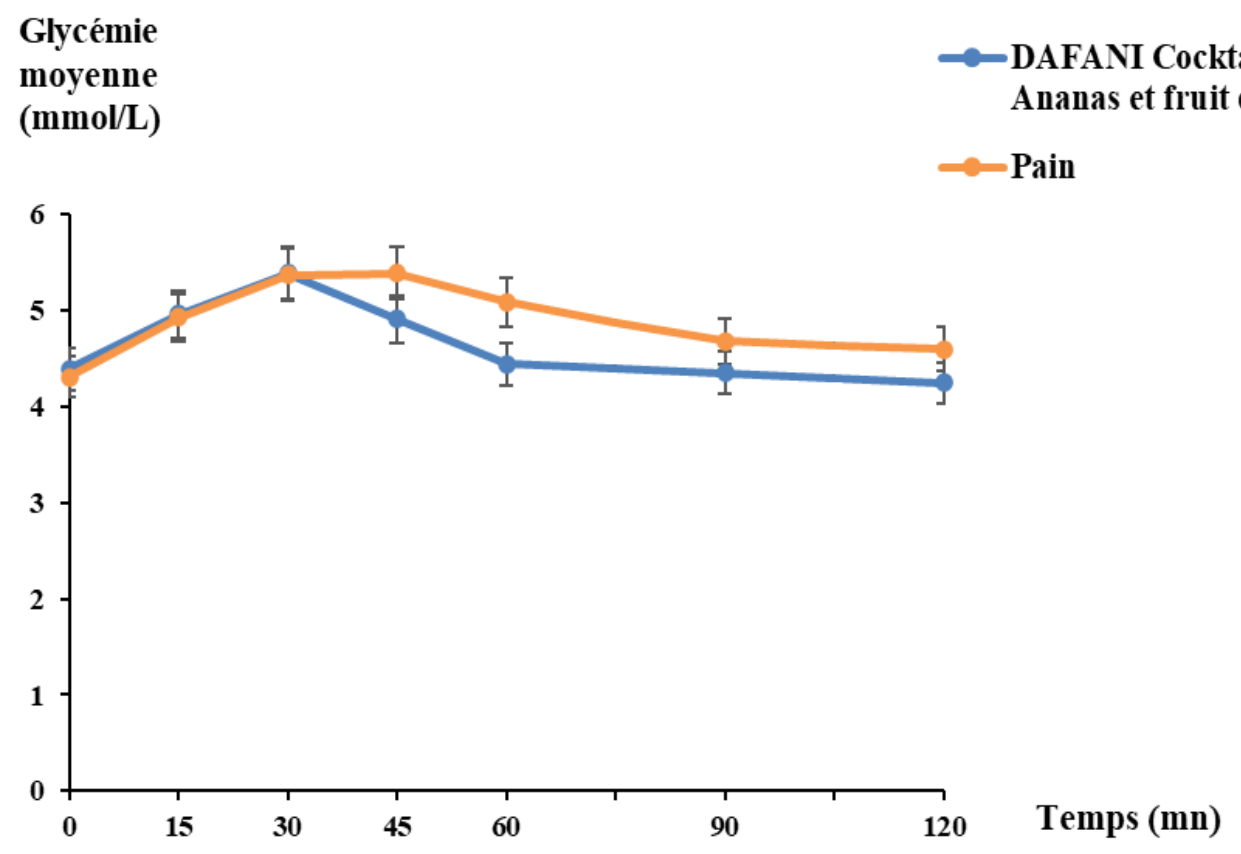

Figure 1C : Groupe 3 (Jus DAFANI cocktail mangue-ananas-fruit de la passion).

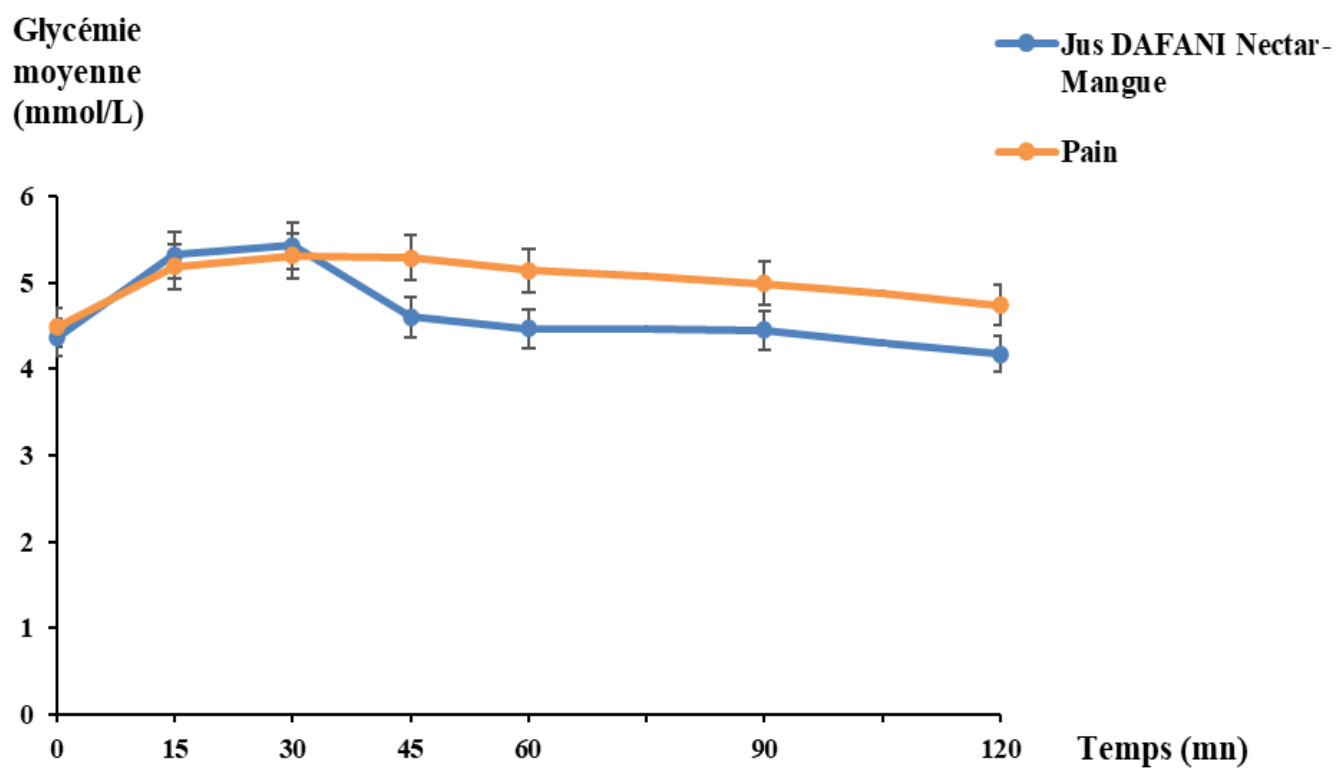

Figure 1D : Groupe 4 (Jus DAFANI nectar mangue).

Figure 1 : Evolution de la glycémie moyenne en fonction du temps. 
Tableau 2 : Index glycémique (IG) et charge glycémique (CG) des jus de fruits testés.

\begin{tabular}{lcc}
\hline & IG $(\%)$ & CG \\
\hline DAFANI orange-mangue & $47,50 \pm 27,55$ & $11,87 \pm 2,52$ \\
Jus d'ananas IRA & $64,12 \pm 23,74$ & $16,03 \pm 2,16$ \\
DAFANI cocktail MAP & $62,25 \pm 33,58$ & $11,47 \pm 3,15$ \\
DAFANI nectar mangue & $87,62 \pm 64,91$ & $15,56 \pm 6,04$ \\
\hline
\end{tabular}

MAP : mangue-ananas-fruit de la passion.

\section{DISCUSSION}

La réponse glycémique induite par la consommation des aliments varie selon le type d'aliment. Dans cette étude, la réponse glycémique des jus de fruits testés était inférieure à celle de l'aliment de référence comme rapporté par Freitas et al. (2021). Dans l'étude de Freitas et al. (2021), le jus de citron a entrainé un pic glycémique à T60 min. Le jus de pomme a donné une réponse glycémique qui a culminé à T45 min (Laue et al., 2019). La variabilité des pics et réponses glycémiques peut être due, en dehors des différences méthodologiques, à la teneur variable en nutriments des fruits ayant servi à la fabrication des jus (Ballance et al., 2018). En effet, les aléas climatiques et le type de climat de chaque milieu sur les fruits (degré de murissement), les types de cultivar, les variétés de fruits et le type de sol utilisé pour la culture sont autant de facteurs qui peuvent modifier la composition en nutriments d'un fruit (Ballance et al., 2018).

Les IG obtenus dans cette étude variaient en fonction du jus testé. La nature et la composition des fruits ayant servi à la fabrication de ces jus pourraient expliquer cette observation. Plusieurs facteurs, liés à la composition des fruits, sont susceptibles de modifier la réponse glycémique, comme la nature des oses (fructose, glucose, saccharose), la nature de l'amidon (amylose, amylopectine, interaction entre amidon et autres nutriments, résistance de l'amidon), les fibres alimentaires
(Bosch-Sierra et al., 2019). D'autres composants alimentaires (graisses, protéines, anti nutriments, anti oxydants et acides organiques) et les composés phénoliques (flavonoïdes, tanins...) présents dans les végétaux dont les fruits ont des effets sur la réponse glycémique (Koutouan et al., 2019). Ces nutriments retrouvés dans les fruits sont également présents dans les jus de fruits mais en quantité réduite en rapport avec le processus de fabrication de ces jus. Certains de ces nutriments comme les flavonoïdes, les fibres alimentaires et les acides organiques ont un effet hypoglycémiant. L'effet hypoglycémiant des flavonones et flavonoïdes des jus de fruits peut être dû à plusieurs mécanismes (Liu et al., 2006 ; Cazarolli et al., 2008 ; Koutouan et al., 2019): (i) ils inhibent l' $\alpha$-glucosidase au niveau intestinal réduisant donc la disponibilité de molécules de glucose libre pour leur absorption ; (ii) par inhibition compétitive du transporteur de glucose dépendant du sodium (SGLT), ils empêchent l'absorption intestinale du glucose ; (iii) ils ont également la capacité de stimuler la sécrétion de l'insuline en intervenant directement sur les cellules $\beta$ du pancréas, par activation de la cascade de signalisation cAMP/PKA conduisant à leur effet insulinotrope. Certaines études ont montré que les fibres alimentaires contenues dans les aliments ont pour propriétés de ralentir la digestion et donc diminuent, par ce mécanisme, la réponse glycémique (Liu et al., 
2006). En effet, les fibres alimentaires, constituants endogènes de la substance végétale du régime alimentaire, constituées des polysaccharides non amylacés et de lignine ne contenant aucune liaison $\alpha$-glucosidique (Liu et al., 2006), résistent à la digestion enzymatique chez les êtres humains. A teneur en glucides égale, les IG des jus de fruits testés étaient différents. D'autres constituants de ces jus de fruits pourraient en être la base. En effet, les jus fabriqués à base d'orange pure (Citrus sinensis) sont riches en acides organiques. Ces acides, en ralentissant la vidange gastrique, limitent l'absorption rapide des glucides du bol alimentaire et réduisent ainsi la réponse glycémique. Les acides organiques peuvent en outre réduire la glycémie par inhibition de l' $\alpha$ amylase (Fred-Jaiyesimi et al., 2017 ; Freitas et Feunteun, 2018). La teneur variable en acides organiques des jus de fruits étudiés pourrait expliquer la variabilité observée au niveau des IG.

Tous les jus de fruits utilisés avaient une CG modérée (entre 11 et 19), témoignant de leur effet hyperglycémiant modéré. A teneur en glucides égale (13 g environ) et IG différents, la $C G$ des quatre jus de fruits était modérée. Au cours des dernières décennies, un certain nombre d'études, tant observationnelles qu'interventionnelles, ont été menées concernant l'association IG et CG avec différents aspects des maladies métaboliques et le risque cardiovasculaire. Bien que certains résultats aient été favorables, en particulier chez les patients diabétiques et ceux présentant une insulino-résistance (Fan et al., 2012), d'autres études n'ont pas montré des avantages importants (Shahdadian et al., 2019). Une association positive entre l'IG et le risque de diabète de type 2 ainsi que la notion de consommation d'aliments à IG faible par les patients diabétiques comme régime alimentaire sain a été soutenue par l'étude prospective à grande échelle « Nurses' Health Study and the Health Professionals Follow-Up Study » (Hodge et al., 2004); cependant, des études plus récentes telles que le «Atherosclerosis Risk in Communities Study (ARIC) » (Hardy et al., 2010) et les travaux de Sluijs et al. (2013), n'ont pas montré de relation entre l'IG ou la CG et des aspects du contrôle de la glycémie. Une méta-analyse de Shahdadian et al. (2019) n'a montré aucune association significative entre l'IG ou la CG alimentaire et la mortalité par maladie cardiovasculaire (MCV). En revanche, Fan et al. (2012) ont démontré une association positive significative entre un IG et une CG élevés et les maladies coronariennes. Dans l'ensemble, les données concernant l'association entre IG/CG et les événements cardiovasculaires peuvent être considérées comme équivoques.

La principale limite de ce travail était l'utilisation des valeurs nutritives inscrites sur les emballages des jus de fruits pour le calcul des quantités équivalentes de glucides données aux sujets. Leur détermination permettrait de vérifier les déclarations des fabricants de ces jus de fruits.

\section{Conclusion}

Il ressort de cette étude que la réponse glycémique des jus testés était inférieure à celle des aliments de référence que sont le glucose et le pain blanc. L'IG du jus DAFANI cocktail orange-mangue était faible, ceux des jus d'ananas IRA et DAFANI cocktail mangueananas-fruit de la passion étaient moyens, tandis que le jus DAFANI nectar mangue avait un IG élevé. Tous les jus de fruits testés avaient une CG modérée. Bien que la CG des jus de fruits étudiés soit modérée, leur consommation contrôlée en particulier chez les sujets diabétiques est nécessaire.

\section{CONFLIT D'INTERETS}

Les auteurs déclarent qu'ils n'ont aucun conflit d'intérêts pour cet article.

\section{CONTRIBUTIONS DES AUTEURS}

MG a conçu l'étude, supervisé la collecte et l'analyse des données, et écrit le 
draft du manuscrit. GS a écrit le protocole et fait la collecte et l'analyse des données. ST, $\mathrm{AA}$ et IMC ont participé à la collecte des données et fait la revue de littérature. LB-M et SA ont validé la version finale du manuscrit.

\section{REMERCIEMENTS}

Les auteurs remercient les volontaires qui ont participé à l'expérimentation de la présente étude.

\section{REFERENCES}

Augustin LSA, Kendall CWC, Jenkins DJA, Willett WC, Astrup A, Barclay AW, Björck I, Brand-Miller JC, Brighenti F, Buyken AE, Ceriello A, La Vecchia C, Livesey G, Liu S, Riccardi G, Rizkalla SW, Sievenpiper JL, Trichopoulou A, Wolever TMS, Baer-Sinnott S, Poli A. 2015. Glycemic index, glycemic load and glycemic response: An Internantional Scientific Consensus Summit from the International Carbohydrate Qualty Consortium (ICQC). Nutr. Metab. Cardiovasc. Dis., 25(9): 795-815. DOI:10.1016/j.numecd.2015.05.005

Ballance S, Knutsen SH, Fosvold ØW, Fernandez AS, Monro J. 2018. Predicting mixed-meal measured glycaemic index in healthy subjects. Eur. J. Nutr., 58(7): 2657-2667. DOI : 10.1007/s00394-018$1813-\mathrm{Z}$

Bosch-Sierra N, Marqués-Cardete R, GurreaMartínez A, Grau-Del Valle C, Morillas C, Hernández-Mijares A, Bañuls C. 2019. Effect of Fibre-Enriched Orange Juice on Postprandial Glycaemic Response and Satiety in Healthy Individuals: An Acute, Randomised, Placebo-Controlled, Double-Blind, Crossover Study. Nutrients, 11(12): $3014 . \quad$ DOI: 10.3390/nu11123014

Brouns F, Bjorck I, Frayn KN, Gibbs AL, Lang V, Slama G, Wolever TMS. 2005. Glycaemic index methodology.
Nutr. Res. Rev., 18: 145-171. DOI: 10.1079/NRR2005100

Cazarolli LH, Zanatta L, Alberton EH, Santos Reis Bonorino Figueiredo M, Folador P, Damazio RG, Pizzolatti MG, Mena Barreto Silva FR. 2008. Flavonoids: cellular and molecular mechanism of action in glucose homeostasis. Mini-Rev. Med. Chem., 8(10): 1032-1038. DOI: 10.2174 / 138955708785740580

Fan J, Song Y, Wang Y, Hui R, Zhang W. 2012. Dietary glycemic index, glycemic load, and risk of coronary heart disease, stroke, and stroke mortality: A systematic review with meta-analysis. Plos One, 7(12): e52182. DOI: 10.1371/journal.pone.0052182

FAO/WHO. 1998. Carbohydrates in human nutrition. Report of a joint FAO/WHO expert consultation. FAO Food Nutr. Pap., 66:1-140.

Foster-Powell K, Holt SHA, Brand-Miller JC. 2002. International table of glycemic index and glycemic load values: 2002. Am. J. Clin. Nutr., 76(1): 5-56. DOI: 10.1093/ajcn/76.1.5

Fred-Jaiyesimi AA, Wilkins RM, Abo KA. 2017. Glucose lowering activities of mombintane I and mombintane II isolated from the leaves of Spondias mombin L. Int. J. Biol. Chem. Sci., 11(3): 1315-1319. DOI: 10.4314/ijbcs.v11i3.31

Freitas D, Boué F, Benallaoua M, Airinei G, Benamouzig R, Le Feunteun S. 2021. Lemon juice, but not tea, reduces the glycemic response to bread in healthy volunteers: a randomized crossover trial. Eur. J. Nutr., 60(1):113-122. DOI: 10.1007/s00394-020-02228-x

Freitas D, Le Feunteun S. 2018. Acid induced reduction of the glycaemic response to starch-rich foods: the salivary $\alpha$-amylase inhibition hypothesis. Food Funct., 9(10): 5096-5102. DOI :10.1039/c8fo01489b

Gomina Assoumanou M, Saka DH, Akpona SA. 2011. Réponse glycémique induite 
par quatre boissons alcoolisées locales du Bénin chez des sujets adultes jeunes sains. Int. J. Biol. Chem. Sci., 5(3): 11531164.

Hardy DS, Hoelscher DM, Aragaki C, Stevens J, Steffen LM, Pankow JS, Boerwinkle E. 2010. Association of glycemic index and glycemic load with risk of incident coronary heart disease among Whites and African Americans with and without type 2 diabetes: The Atherosclerosis Risk in Communities study. Ann. Epidemiol., 20(8): 610-616. DOI: 10.1016/j.annepidem.2010.05.008

Hodge AM, English DR, O'Dea K, Giles GG. 2004. Glycemic index and dietary fiber and the risk of type 2 diabetes. Diabetes Care, 27(11): 2701-2706. DOI: 10.2337/diacare.27.11.2701

Kimokoti RW, Millen BE. 2016. Nutrition for the Prevention of Chronic Diseases. Med. Clin. North. Am., 100(6): 1185-1198. DOI: 10.1016/j.mcna.2016.06.003

Koutouan FP, Yapi YM, Wandan EN, Bodji NC, N'da KP. 2019. Composition en polyphénols totaux et en tanins des feuilles de neuf variétés de Cajanus cajan (L.) Millsp. au cours du premier cycle de croissance et en fonction du mode d'exploitation. Int. J. Biol. Chem. Sci., 13(2): $\quad 882-898$. DOI: 10.4314/ijbcs.v13i2.25

Laue C, Balance S, Knutsen SH, Papazova E, Soeth E, Pannenbeckers A, Schrezenmeir J. 2019. Glycemic response to low sugar apple juice treated with invertase, glucose oxidase and catalase. Eur. J. Clin. Nutr., 73(10): 1382-1391. DOI: 10.1038/s41430-019-0421-1

Liu D, Zhen W, Yang Z, Carter JD, Si H, Reynolds KA. 2006. Genistein Acutely Stimulates Insulin Secretion in Pancreatic $\beta$-Cells Through a cAMP-Dependent Protein Kinase Pathway. Diabetes, 55(4): 1043-1050.

DOI: 10.2337/diabetes.55.04.06.db05-1089
Madhu SV. 2017. Glycaemic index: challenges in translating concept to practice. Int. J. Diabetes Dev. Ctries, 37(4): 377-378. DOI : 10.1007/s13410017-0585-y

Makoutode A, Saizonou J, Glele Ahanhanzo Y, Sossa CJ, Agueh V. 2017. Prévalence et facteurs associés à la surcharge pondérale chez les adolescents dans une ville secondaire au Bénin. Int. J. Biol. Chem. Sci., 11(2): 798-805. DOI: 10.4314/ijbcs.v11i2.21

Monro JA, Shaw M. 2008. Glycemic impact, glycemic glucose equivalents, glycemic index, and glycemic load: definitions, distinctions, and implications. Am. J. Clin. Nutr., 87(1): 237S-243S. DOI: 10.1093/ajcn/87.1.237S

Muraki I, Imamura F, Manson JAE, Hu FB, Willett WC, van Dam RM, Qi S. 2013. Fruit consumption and risk of type 2 diabetes: results from three prospective longitudinal cohort studies. BMJ., 347. DOI: 10.1136/bmj.f5001

Murphy MM, Barrett EC, Bresnahan KA, Barraj LM. 2017. 100\% Fruit juice and measures of glucose control and insulin sensitivity: a systematic review and metaanalysis of randomised controlled trials. J. Nutr. Sci., 6(59): 1-15. DOI:10.1017/jns.2017.63

Papandreou D, Magriplis E, Abboud M, Taha Z, Karavolia E, Karavolias C, Zampelas A. 2019. Consumption of Raw Orange, 100\% Fresh Orange Juice, and NectarSweetened Orange Juice-Effects on Blood Glucose and Insulin Levels on Healthy Subjects. Nutrients, 11(9): 2171. DOI: $10.3390 /$ nu11092171

Pereira MA, Fulgoni VL III. 2010. Consumption of $100 \%$ fruit juice and risk of obesity and metabolic syndrome: findings from the national health and nutrition examination survey 1999-2004. J. Am. Coll. Nutr., 29(6): 625-629. DOI: 10.1080/07315724.2010.10719901 
Shahdadian F, Saneei P, Milajerdi A, Esmaillzadeh A. 2019. Dietary glycemic index, glycemic load, and risk of mortality from all causes and cardiovascular diseases: A systematic review and dose-response meta-analysis of prospective cohort studies. Am. J. Clin. Nutr., 110(4): 921-937. DOI: 10.1093/ajen/nqz061

Sluijs I, Beulens JWJ, van der Schouw YT, van der A DL, Buckland G, Kuijsten A, Schulze MB, Amiano P, Ardanaz E, Balkau B, Boeing H, Gavrila D, Grote VA, Key TJ, Li K, Nilsson P, Overvad K, Palli D, Panico S, Quirós JR, Rolandsson O, Roswall N, Sacerdote C, Sánchez MJ, Sieri S, Slimani N, Spijkerman AMW, Tjønneland A, Tumino R, Sharp SJ, Langenberg C, Feskens EJM, Forouhi NG, Riboli E, Wareham NJ, InterAct consortium. 2013. Dietary glycemic index, glycemic load, and digestible carbohydrate intake are not associated with risk of type 2 diabetes in eight European countries. $J$.
Nutr., 143(1): 93-99. $\quad$ DOI:

10.3945/jn.112.165605

Tsilas CS, de Souza RJ, Mejia SB, Mirrahimi A, Cozma AI, Jayalath VH, Ha V, Tawfik R, Di Buono M, Jenkins AL, Leiter LA, Wolever TMS, Beyene J, Khan $\mathrm{T}$, Kendall CWC, Jenkins DJA, Sievenpiper JL. 2017. Relation of total sugars, fructose and sucrose with incident type 2 diabetes: A systematic review and meta-analysis of prospective cohort studies. CMAJ., 189(20): E711E720. DOI: 10.1503/cmaj.160706

Wang YC, Bleich SN, Gortmaker SL. 2008. Increasing caloric contribution from sugar-sweetened beverages and 100\% fruit juices among US children and adolescents, 1988-2004. Pediatrics, 121: e1604-e1614. DOI : 10.1542/peds.20072834

Xi B, Li S, Liu Z, Tian H, Yin X, Huai P, Tang W, Zhou D, Steffen LM. 2014. Intake of Fruit Juice and Incidence of Type 2 Diabetes: A Systematic Review and Meta-Analysis. Plos One, 9(3): e93471. DOI: 10.1371/journal.pone.0093471 